\begin{tabular}{|c|c|}
\hline Title & $\begin{array}{l}\text { Di st urb- Free Witing Oper at i on for Fer roel ectric- } \\
\text { Gate Fi el d- Effect Transi st or Nemori es wi th } \\
\text { I nt er medi at e El ectrodes }\end{array}$ \\
\hline Author(s) & Hor i ta, Susumi; Tri nh, Bui Nguyen Quoc \\
\hline Citation & $\begin{array}{l}\text { I EEE Transact } i \text { ons on El ectron Devi ces, 56(12): } \\
\text { 3090-3096 }\end{array}$ \\
\hline Issue Date & 2009- 12 \\
\hline Type & Journal Arti cle \\
\hline Text version & publ i sher \\
\hline URL & ht t p: //hdl . handl e. net /10119/8476 \\
\hline Rights & 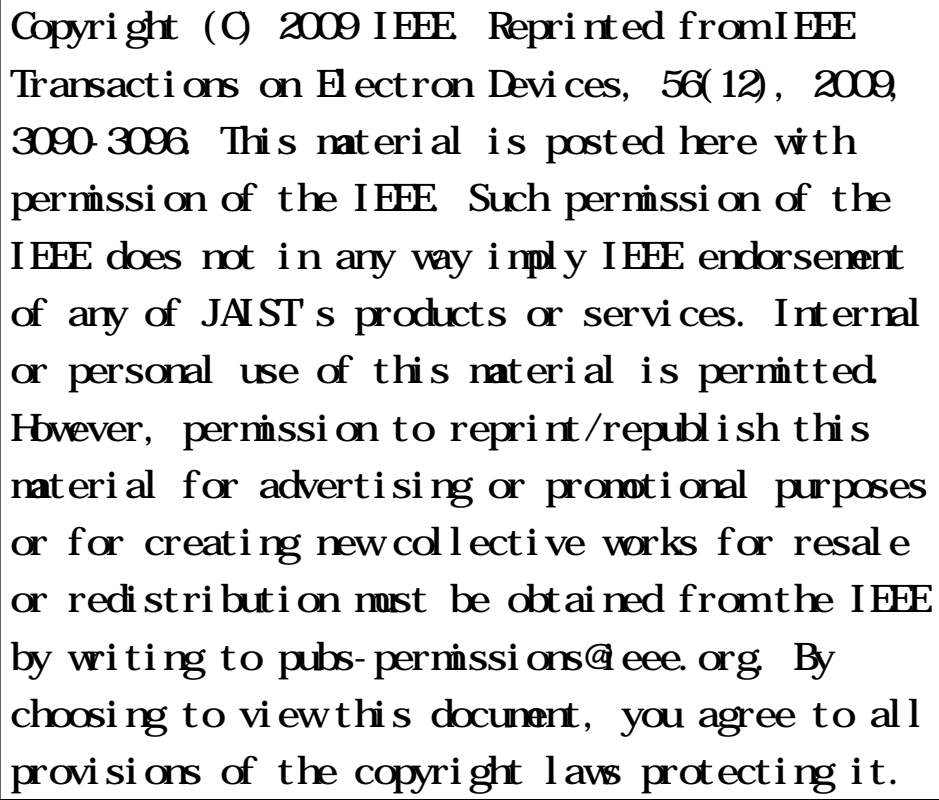 \\
\hline Description & \\
\hline
\end{tabular}

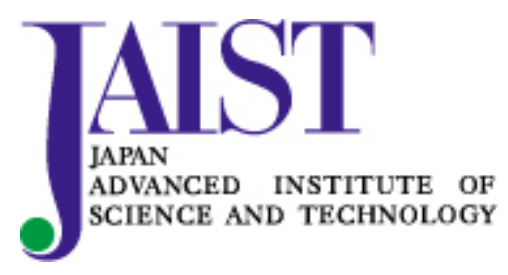




\title{
Disturb-Free Writing Operation for Ferroelectric-Gate Field-Effect Transistor Memories With Intermediate Electrodes
}

\author{
Susumu Horita and Bui Nguyen Quoc Trinh
}

\begin{abstract}
To achieve disturb-free writing, we proposed a new writing operation for ferroelectric-gate field-effect transistor memories with intermediate electrodes. The writing voltages $V_{W}$ applied to the wordlines for $\mathrm{Pr}^{+}$and $\mathrm{Pr}^{0}$ memory states are the same pulse magnitudes, which consist of $V_{W}^{+}$followed by $V_{W}^{-}$, whereas the bias timings of the bitline voltages differ from each other. The bitline voltage for the $\mathrm{Pr}^{+}$memory state is set high when $V_{W}$ is set $V_{W}^{+}$, and it is set to low by the time when $V_{W}$ is changed to $V_{W}^{-}$. On the other hand, the bitline voltage for the $\operatorname{Pr}^{0}$ memory state is set high until the whole writing pulse of $\left(V_{W}^{+}+V_{W}^{-}\right)$is finished. This is verified experimentally using a discrete circuit, which showed that the new writing operation achieves disturb-free writing. The memory consists of two transistors for data writing and reading. With the obtained experimental results, we discuss the possibilities of high integration of this memory as well as low reading voltage.
\end{abstract}

Index Terms-Disturb free, ferroelectric-gate memory, ferroelectric memory, nondestructive readout, write disturbance.

\section{INTRODUCTION}

$\mathbf{F}$ ERROELECTRIC-GATE field-effect transistor (F-FET) memories are known to be one of the ultimate nonvolatile memories because of their remarkable features, such as nondestructive readout, high packing density, high reading speed, and low power consumption [1], [2], compared with ferroelectric random-access memories (FeRAMs). Furthermore, they have the advantages of low writing voltage, fast writing speed, and high endurance, compared with flash memories [3]. Although considerable researches on F-FET memories have been carried out [4]-[7], F-FET memories have not been commercialized because of problems such as short retention time [8], [9] and high writing voltage [10], [11]. To overcome these problems, we have proposed a new F-FET memory (denoted as IF-FET memory), in which an intermediate electrode (IE) for data writing is inserted between the ferroelectric capacitor $C_{f}$ and the buffer layer (the gate) of a reading MOSFET (R-MOSFET) [13]-[15]. For data writing, using the IE, a writing voltage

Manuscript received January 27, 2009; revised September 4, 2009. First published October 30, 2009; current version published November 20, 2009. The review of this paper was arranged by Editor D. Esseni.

The authors are with the Graduate School of Materials Science, Japan Advanced Institute of Science and Technology, Nomi 923-1292, Japan (e-mail: horita@jaist.ac.jp).

Color versions of one or more of the figures in this paper are available online at http://ieeexplore.ieee.org.

Digital Object Identifier 10.1109/TED.2009.2032744
$V_{W}$ is applied directly to the $C_{f}$ without voltage dropping on the buffer layer, and for data reading, a reading voltage $V_{R}$ is applied between the top of $C_{f}$ and the source of R-MOSFET with the IE being electrically floated. The memory needs another MOSFET for data writing in addition to R-MOSFET. The detailed configuration is given in Section II. Recently, we have also proposed an improved reading operation for perfect nondestructive readout [16]. In this operation, we used two kinds of memory states, a positive remanentpolarization $\mathrm{Pr}^{+}$state and a nearly nonpolarized $\operatorname{Pr}^{0}$ state in $C_{f}$. For data reading, following a positive reading pulse with the magnitude $V_{R}^{+}$to decode the memory state of $C_{f}$, a negative reading pulse with the magnitude $V_{R}^{-}$is applied to return the readout memory state to the initial one. It was confirmed experimentally that this reading operation improved the readout endurance more than $10^{8}$ reading cycles and the retention time to exceed ten years [16], using a discrete circuit of IF-FET memory or one memory cell. It can be expected that the nondestructive readout by this operation endures until ferroelectric properties of $C_{f}$ are degraded intrinsically such as fatigue and imprint. High endurance is another advantage of IF-FET over 1-transistor-1-capacitor (1T-1C) FeRAM in addition to the previously mentioned advantages of F-FET, except for that with high packing density. The high endurance results from the use of minor polarization-electric field $(P-E)$ loops for data reading instead of full switching of polarization like that in FeRAM, which is explained in detail later. Ishiwara et al. proposed a 1-transistor-2-capacitor (1T-2C), whose operation principle is similar to IF-FET, as a substitute of conventional F-FET. The total area of $2 \mathrm{C}$ in $1 \mathrm{~T}-2 \mathrm{C}$ cannot be reduced to and overlapped with the gate area of R-MOSFET, which is not suitable for high integration of the memory. On the contrary, $C_{f}$ of IF-FET can be fabricated just on the gate with the same area. The other comment on comparison with $1 \mathrm{~T}-2 \mathrm{C}$ has been mentioned before [12].

However, disturbance of data in the writing operation (write disturbance) of IF-FET occurs in the case of memory array structure of an integrated circuit, which is a serious problem for practical use. We propose a new writing operation of IF-FET after demonstrating the problem related to a previous writing operation. By using a discrete circuit, we have also verified experimentally that the new writing operation is write disturb free as compared with the previous one. Finally, with the obtained results, we discuss the integration of IF-FET and the related issue of reading voltage. 


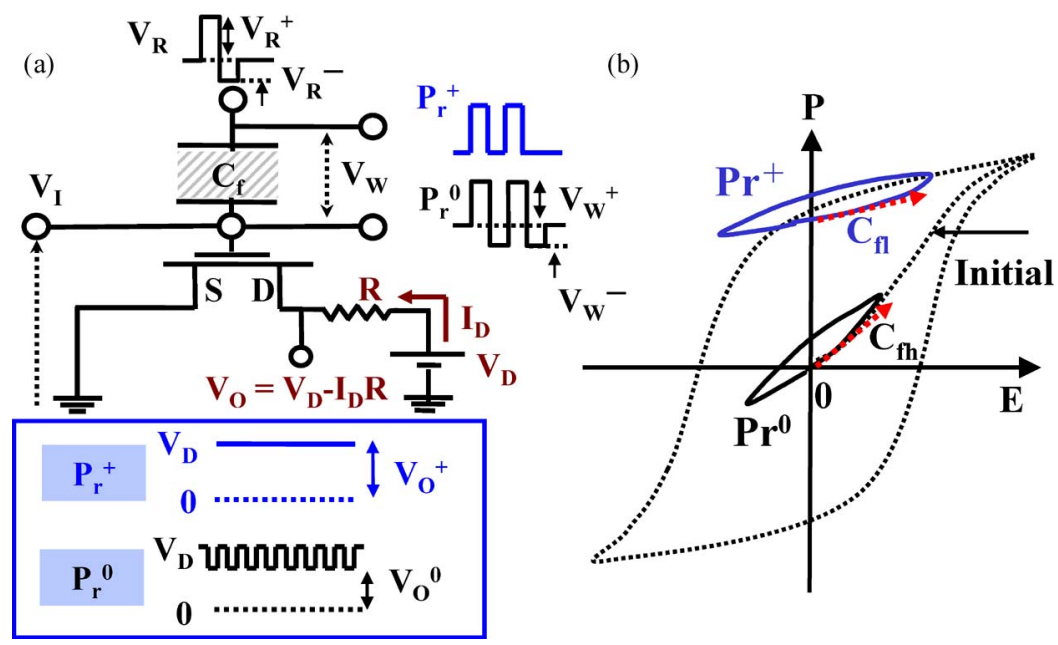

Fig. 1. (a) (Top) Schematic equivalent circuit of the IF-FET memory, and (bottom) output voltages $V_{O}^{+}$and $V_{O}^{0}$ for $\operatorname{Pr}^{+}$and $\operatorname{Pr}^{0}$ memory states, respectively, corresponding to the continuous reading pulse $V_{R}$. (b) Schematic $P-E$ hysteresis loop and initial curve (dotted lines) of a ferroelectric capacitor $C_{f}$. (Solid lines) Minor loops that originate from $\operatorname{Pr}^{+}$and $\operatorname{Pr}^{0}$ are traces of $P-E$ in data reading. The $C_{\mathrm{fl}}$ and $C_{\mathrm{fh}}$ are the capacitance values of $\operatorname{Pr}^{+}$and $\operatorname{Pr}^{0}$ states, respectively, in the positive $E$.

\section{Problem And Improvement of Data Disturbance}

Fig. 1(a) shows a schematic equivalent circuit of IF-FET memory, which consists of a ferroelectric capacitor $C_{f}$ connected serially to an n-channel R-MOSFET. In this figure, an output voltage $V_{O}$ is measured on a resistor $R$ at the drain of the MOSFET, which is biased by a de voltage $V_{D}$. As memory states, we use a positive remanent polarization $\mathrm{Pr}^{+}$and a nearly nonpolarized $\operatorname{Pr}^{0}$. Fig. 1(b) shows not only the schematic $P-E$ hysteresis loop and initial $P-E$ curve (dotted lines) but also the two minor loops (solid lines). The minor loops represent the $P-E$ traces during IF-FET reading operation for the $\mathrm{Pr}^{+}$ and $\operatorname{Pr}^{0}$ memory states. $C_{\mathrm{f}}$ and $C_{\mathrm{fh}}$ are denoted as ferroelectric capacitances of $\operatorname{Pr}^{+}$state and $\operatorname{Pr}^{0}$ state, respectively, which are values in response to the positive rising pulses for data reading. Because $C_{f}$ is proportional to the gradient of the $P-E$ slope, the $C_{\mathrm{fh}}$ of $\operatorname{Pr}^{0}$ is larger than the $C_{\mathrm{fl}}$ of $\mathrm{Pr}^{+}$. The outline of the previous operation is as follows: Both memory states are produced by applying writing voltages directly to the $C_{f}$, where the drains are grounded at $V_{D}=0$. The writing voltage $V_{W}$ for $\mathrm{Pr}^{+}$state was a positive square pulse, and $V_{W}$ for $\operatorname{Pr}^{0}$ state was a combined square pulse consisting of a positive pulse magnitude $V_{W}^{+}$followed by a negative pulse magnitude $V_{W}^{-}$, as shown in Fig. 1(a). $V_{W}^{+}$is to reset the previous stored memory state, and $V_{W}^{-}$is to determine the polarization of $\operatorname{Pr}^{0}$ state. For data reading, a reading voltage consisting of a positive pulse magnitude $V_{R}^{+}$followed by a negative pulse magnitude $V_{R}^{-}$is applied between the top of $C_{f}$ and the ground, where the IE is electrically floated and the drain is biased by a positive $V_{D}$. Therefore, the reading voltage $V_{R}$ is applied to $C_{f}$ and $C_{i}$ in series, and it is divided into two voltage drops of $V_{f}$ on $C_{f}$ and $V_{I}$ on $C_{i}$, where $C_{i}$ is an input capacitor of the R-MOSFET. Because $V_{I}$ or IE voltage of $\mathrm{Pr}^{+}$state is lower than that of $\operatorname{Pr}^{0}$ state, which is due to $C_{\mathrm{fl}}<C_{\mathrm{fh}}$, a drain-current $I_{D}$ of the R-MOSFET in the $\mathrm{Pr}^{+}$state is much smaller than that in the $\operatorname{Pr}^{0}$ state. The reading $V_{R}^{+}$is required not to be as high as to saturate polarization like FeRAM but to produce a detectable difference in $I_{D}$. The memory states can be distinguished by

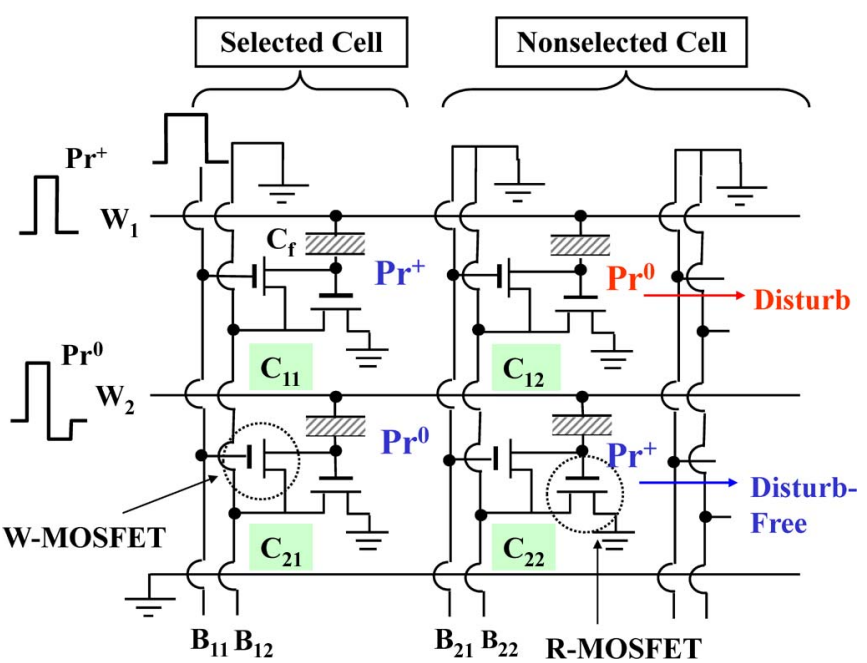

Fig. 2. Previous writing operation for an integrated array of IF-FET memory cells. A writing voltage for $\mathrm{Pr}^{+}$memory state is a positive pulse and that for $\operatorname{Pr}^{0}$ memory state is a combined pulse consisting of a positive pulse followed by a smaller negative pulse. For data writing, the W-MOSFETs in the selected cells are turned on to apply the writing voltages directly to the $C_{f}$. This operation causes write disturbance of $\mathrm{Pr}^{0}$ memory states because the $\mathrm{Pr}^{+}$ writing voltage is positive.

measuring a difference in $V_{O}=V_{D}-I_{D} \mathrm{R}, \Delta V_{O}=V_{O}^{+}-$ $V_{O}^{0}$, between $V_{O}^{+}$for $\operatorname{Pr}^{+}$state and $V_{O}^{0}$ for $\operatorname{Pr}^{0}$ state.

Figs. 2 and 3 show the schematic arrays of IF-FET memory cells for the previous writing operation and the proposed writing operation, respectively, in integrated circuits. In each memory cell, an additional writing MOSFET (W-MOSFET) is used as a switch to short-circuit the R-MOSFET to the ground for data writing. The W-MOSFETs are integrated for the selection of writing cells. For large-scale integration, this configuration seems unsuitable because a $1 \mathrm{C}-2 \mathrm{~T}$ occupies a large area. However, in an actual integrated circuit, the capacitor can be fabricated just over the gate of R-MOSFET, the area being the same as the gate. Therefore, the configuration of IF-FET memory consists of two transistors (2T memory) in 


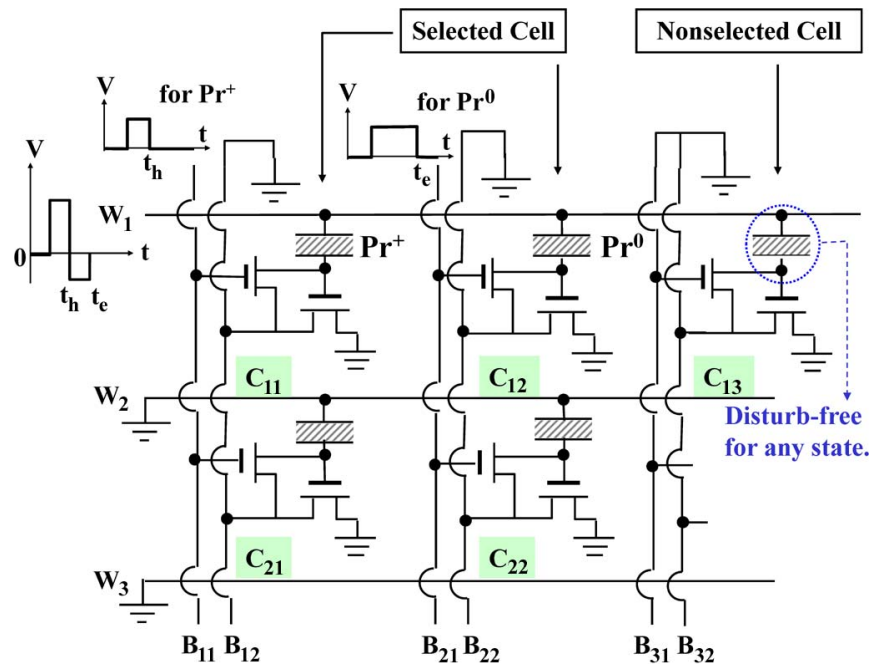

Fig. 3. Proposed writing operation for an integrated array of IF-FET memory cells. The writing voltages to the wordlines for $\mathrm{Pr}^{+}$and $\mathrm{Pr}^{0}$ memory states are the same. They are combined pulses consisting of a positive pulse magnitude $V_{W}^{+}$followed by a negative pulse magnitude $V_{W}^{-}$, where the switching time from $V_{W}^{+}$to $V_{W}^{-}$is $t_{h}$ and the end time of the writing voltage is $t_{e}$. The bitline switching times from high to low for the $\operatorname{Pr}^{+}$and $\operatorname{Pr}^{0}$ states are before $t_{h}$ and after $t_{e}$, respectively. Using this operation, write disturb free is achieved.

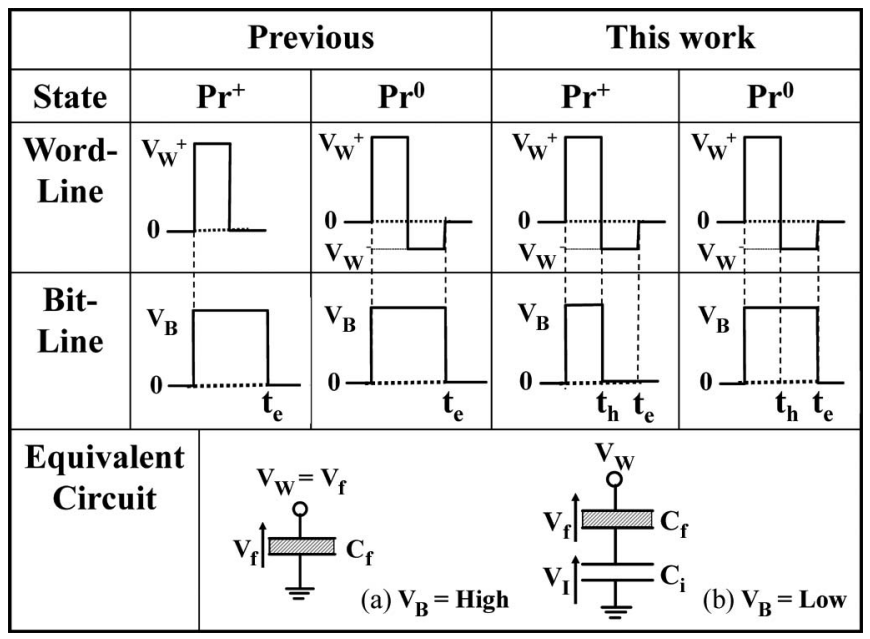

Fig. 4. Comparison of bias timings in wordline and bitline between the previous and proposed writing operations, for $\operatorname{Pr}^{+}$and $\operatorname{Pr}^{0}$ memory states. The bottom shows the equivalent circuits of the memory cells for (a) high bitline and (b) low bitline in writing operation. $t_{e}$ and $t_{h}$ are explained in the caption of Fig. 3.

practice. This issue is further discussed later. Fig. 4 also shows the comparison of bias timings of wordline and bitline pulses between the previous and the proposed writing operations, for $\mathrm{Pr}^{+}$and $\mathrm{Pr}^{0}$ memory states. In the previous case, the wordline pulses for $\mathrm{Pr}^{+}$and $\mathrm{Pr}^{0}$ states are different from each other, and the bitline pulses are the same but vice versa in the proposed case. The bottom figures in Fig. 4(a) and (b) show the equivalent circuits for high and low bitline voltages, respectively, of the memory cells in writing operation. When bitlines are high, the W-MOSFETs are ON-state, and the writing voltages are applied directly to $C_{f}$. When bitlines are low, the W-MOSFETs are OFF-state, and the writing voltages are applied to $C_{f}$ and $C_{i}$ in series, which is similar to a readout case.
The previous writing operation is explained using Figs. 2 and 4. By setting the bitline $B_{11}$ high and applying the writing voltages of $\mathrm{Pr}^{+}$and $\operatorname{Pr}^{0}$ to the wordlines $\mathrm{W}_{1}$ and $\mathrm{W}_{2}$, respectively, the $C_{11}$ and $C_{21}$ cells store $\operatorname{Pr}^{+}$and $\operatorname{Pr}^{0}$ memory states, respectively, where the $\mathrm{B}_{12}$ line is grounded. By this writing operation, the memory cells can be taken as the equivalent circuits of Fig. 4(a). The nonselected cells with the ground bit lines (e.g., the $C_{12}$ of $\operatorname{Pr}^{0}$ and $C_{22}$ of $\mathrm{Pr}^{+}$) are also applied by the writing voltages of $\mathrm{Pr}^{+}$and $\mathrm{Pr}^{0}$, respectively, and some voltages $V_{f}$ drop on the $C_{f}$ 's, as shown in Fig. 4(b) of the equivalent circuit. For the case of $\operatorname{Pr}^{0}$ writing, the $C_{22}$ of $\operatorname{Pr}^{+}$ memory state remains because the waveform of the writing pulse of $\operatorname{Pr}^{0}$ is similar to that of a nondestructive reading voltage. This is write disturb free. However, for the case of $\mathrm{Pr}^{+}$ writing, the memory state of $C_{12}$ changes from the $\operatorname{Pr}^{0}$ state toward a $\mathrm{Pr}^{+}$state because of the application of the positive writing voltage. This is a severe write disturbance. Needless to say, the other disturbances of $\mathrm{Pr}^{+}$state due to $\mathrm{Pr}^{+}$writing and of $\operatorname{Pr}^{0}$ state due to $\operatorname{Pr}^{0}$ writing are free.

To improve write disturbance of $\operatorname{Pr}^{0}$ state due to $\mathrm{Pr}^{+}$writing, we propose a new writing operation. When we write a $\mathrm{Pr}^{+}$ memory state, we use the previous writing voltage for $\operatorname{Pr}^{0}$ instead of $\mathrm{Pr}^{+}$, which is possible by means of controlling bias timing of bitline voltages, as shown in Figs. 3 and 4. In Fig. 3, the operation sequences are explained for the two cases of writing $\operatorname{Pr}^{+}$and $\operatorname{Pr}^{0}$ memory states into the $C_{11}$ and $C_{12}$ cells, respectively. For the $C_{11}$ cell, the high voltage of the $\mathrm{B}_{11}$ line has fallen down to the ground before the writing voltage changes from $V_{W}^{+}$to $V_{W}^{-}$at $t_{h}$. With this operation, the $C_{11}$ cell can be stored as a $\mathrm{Pr}^{+}$state because the positive $V_{W}^{+}$drops on only $C_{f}$ until $t_{h}$, as shown by the equivalent circuit in Fig. 4(a), and then, $V_{W}^{-}$drops on the $C_{f}$ and $C_{i}$ in series until $t_{e}$, as shown by the equivalent circuit in Fig. 4(b). Because the bias condition on the $C_{f}$ and $C_{i}$ is the same as the nondestructive readout case, the $\mathrm{Pr}^{+}$state written by $V_{W}^{+}$remains even after the application of $V_{W}^{-}$. On the other hand, for the $C_{12}$ cell, the voltage of the $\mathrm{B}_{21}$ line does not fall down to the ground until $t_{e}$ in which the whole writing pulse is finished. By this operation, the $C_{12}$ cell can be written into as a $\operatorname{Pr}^{0}$ state. Because the waveform of the writing voltage is the same as the nondestructive reading voltage, the nonselected memory cell (e.g., $\left.C_{13}\right)$ is free from write disturbance.

\section{EXPERIMENTAL SETUP}

We examined the write disturbance using a discrete circuit, as shown in Fig. 1(a). First, a $\mathrm{Pr}^{+}$or $\mathrm{Pr}^{0}$ state was written into the $C_{f}$ by applying the writing voltage between the top and the IE, where the voltage had double periods to produce a steady memory state without writing errors. Next, we disturbed the stored memory by applying writing voltages between the top electrode and the ground continuously, floating the IE electrically. This bias condition could be taken as a writedisturbance situation of a nonselected cell in an integrated circuit. The number of writing times was varied from $10^{0}$ to $10^{8}$, where one time was one period. After that, by measuring $V_{O}$, we estimated the disturbance characteristics of the stored memory state. The sample structure of $C_{f}$ consisted of $\left(\mathrm{RuO}_{x}\right.$ : 


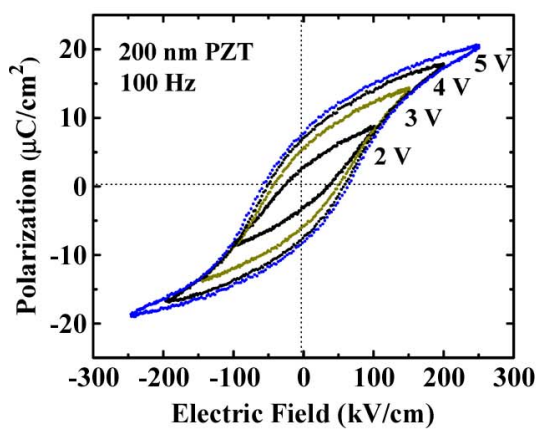

(a)

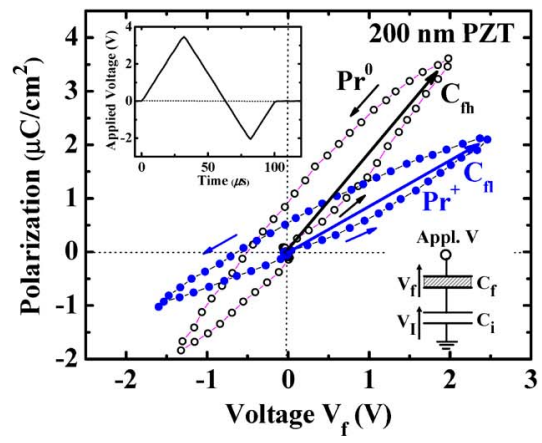

(b)

Fig. 5. (a) $P-E$ hysteresis loops at $100 \mathrm{~Hz}$ of the PZT film used in this paper. (b) Relationships between the polarizations $P$ and applied voltages $V_{f}$ to the PZT film for $\mathrm{Pr}^{+}$and $\operatorname{Pr}^{0}$ memory states in the similar condition of reading operation. The lower inset is the equivalent circuit of Fig. 1 at $V_{D}=2.0 \mathrm{~V}$ for this measurement. The upper inset shows the applied voltage $V$ of a modified triangular waveform. From this, it can be seen that the $C_{\mathrm{fh}}$ is about twice as large as the $C_{\mathrm{fl}}$.

top electrode $) / \mathrm{PtO}_{x} / \mathrm{PZT} / \mathrm{Pt} /\left(\mathrm{RuO}_{x}\right.$ : bottom electrode $)$ on an $\mathrm{SiO}_{2} / \mathrm{Si}$ substrate. The ferroelectric layer was a 200 -nm-thick and highly $(100) /(001)$-oriented PZT film, and the top electrode diameter $D_{t}$ was $100 \mu \mathrm{m}$. The R-MOSFET was a commercial version of 2SK679A with $C_{i}=180 \mathrm{pF}, V_{D}$ was $2 \mathrm{~V}$, and $R$ was $2 \mathrm{k} \Omega$. The pulsewidths of $V_{W}^{+}, V_{W}^{-}, V_{R}^{+}$, and $V_{R}^{-}$were $50 \mu \mathrm{s}$, and the one period for data writing and data reading was $100 \mu \mathrm{s}$. Although it is not necessary for the writing period to be very long in the light of domain switching time and time constant of the measurement circuit, we allowed it to coincide with the reading one.

Fig. 5(a) shows the $P-E$ hysteresis loops of the used PZT film, which were measured by a Sawyer-Tower circuit with a sine wave at $100 \mathrm{~Hz}$. It can be observed that the $P-E$ loops become saturated for applied voltages higher than $4 \mathrm{~V}$. Thus, we set $V_{W}^{+}=4 \mathrm{~V}$, and the $\operatorname{Pr}^{+}$memory state had a positive remanent polarization near $4 \mathrm{~V}$, as shown in Fig. 5(a). For clear readout by application of $V_{R}^{+}$, the gate voltage of the R-MOSFET $V_{I}$ for the $\mathrm{Pr}^{+}$memory state should be equal to or a little smaller than the threshold voltage of the MOSFET, $V_{\mathrm{th}}=1.4 \mathrm{~V}$. Therefore, we set $V_{R}^{+}$to be $3.5 \mathrm{~V}$, taking account of $C_{\mathrm{fh}}, C_{\mathrm{fl}}, C_{i}$, and $V_{\mathrm{th}}$. The actual values of $C_{\mathrm{fh}}$ and $C_{\mathrm{fl}}$ are mentioned later. Application of $V_{W}^{-}$following $V_{W}^{+}$for data writing is to produce a $\operatorname{Pr}^{0}$ memory state from the $\mathrm{Pr}^{+}$state produced by $V_{W}^{+}$. The polarization of the $\operatorname{Pr}^{0}$ should be near the origin of the $P-V$ hysteresis loop from two viewpoints of nondestructive readout and clear difference in output from the $\mathrm{Pr}^{+}$memory state. In addition, application of $V_{R}^{-}$has an important role to return any readout memory state to the initial memory state for the purpose of nondestructive readout. Because the optimum $V_{W}^{-}$and $V_{R}^{-}$depend on the properties of $C_{f}$ and R-MOSFET and are related with each other, we determined them experimentally. Through the process mentioned as in the previous report [16], we set $V_{W}^{+}=4.0 \mathrm{~V}, V_{W}^{-}=-2.3 \mathrm{~V}, V_{R}^{+}=$ $3.5 \mathrm{~V}$, and $V_{R}^{-}=-2.0 \mathrm{~V}$ as the optimum pulse magnitudes in this paper.

Fig. 5(b) shows the relationships between the polarization $P$ of the PZT film and the applied voltage $V_{f}$ to the film for the $\mathrm{Pr}^{+}$and $\operatorname{Pr}^{0}$ memory states. For this measurement, the circuit shown in Fig. 1 was used at $V_{D}=2.0 \mathrm{~V}$, and the equivalent circuit is shown in the lower inset of Fig. 5(b). The applied voltage was a modified triangular waveform as also shown in the upper inset of Fig. 5(b). The maximum and minimum voltages were 3.5 and $-2.1 \mathrm{~V}$, respectively; the duration times of the positive and negative voltages were 62.5 and $37.5 \mu \mathrm{s}$, respectively; and the total or one periodic time was $100 \mu \mathrm{s}$. The maximum and minimum voltages and the total time were matched with the actual reading conditions of $V_{R}^{+}, V_{R}^{-}$, and one period, respectively. The duration times were unintentionally controlled because of the equipment specification, and their exact values are not physically important for this paper. The polarization $P$ was calculated by the formula of $P=4 V_{I}$. $C_{i} /\left(\pi D_{t}^{2}\right)$, and $V_{I}$ was measured by an oscilloscope whose probe had the input resistance and capacitance of $R_{p}=10 \mathrm{M} \Omega$ and $C_{p}=10 \mathrm{pF}$, respectively. Because $C_{i}=180 \mathrm{pF}$ was much larger than $C_{p}$ and the time constant $R_{p}\left(C_{i}+C_{p}\right)=1900 \mu \mathrm{s}$ was much larger than $100 \mu \mathrm{s}$, the input impedance of the oscilloscope can be ignored for this measurement. From this figure, it is shown that the curve slope of the $\mathrm{Pr}^{+}$state is lower than that of the $\operatorname{Pr}^{0}$ state, and the capacitances of the PZT film for the $\operatorname{Pr}^{+}$and $\operatorname{Pr}^{0}$ states are estimated to be $\sim 70$ and $\sim 140 \mathrm{pF}$, respectively. It is also shown that both the $P-V$ curves return to the measurement starting point or the origin after the one periodic measurement, which are similar to the minor loops shown in Fig. 1(b). This implies that nondestructive readout is possible in IF-FET.

To produce $\mathrm{Pr}^{+}$memory states by the new writing operation in a discrete circuit, we added another MOSFET $\left(\mathrm{W}_{d^{-}}\right.$ MOSFET) to the circuit of Fig. 1(a), as shown in the inset of Fig. 6(a). The source, gate, and drain of the $\mathrm{W}_{d}$-MOSFET were connected with the ground, the top electrode of $C_{f}$, and the IE, respectively. When the pulse magnitude of the new writing voltage is a positive $V_{W}^{+}$applied between the top electrode and the ground, the $\mathrm{W}_{d}$-MOSFET is set to the ON-state so that a $\mathrm{Pr}^{+}$state is written into the $C_{f}$. When the pulse magnitude changes to a negative $V_{W}^{-}$, the W-MOSFET was set to the OFFstate so that the $V_{W}^{-}$was applied on the $C_{f}$ and $C_{i}$ in series. By this operation, the $\mathrm{Pr}^{+}$memory state written by $V_{W}^{+}$remains because this writing operation is effectively equivalent to nondestructive readout operation. As a $\mathrm{W}_{d}$-MOSFET, we used the same commercial MOSFET as the R-MOSFET. To write a $\operatorname{Pr}^{0}$ memory state, the writing voltage was applied directly to the $C_{f}$ 


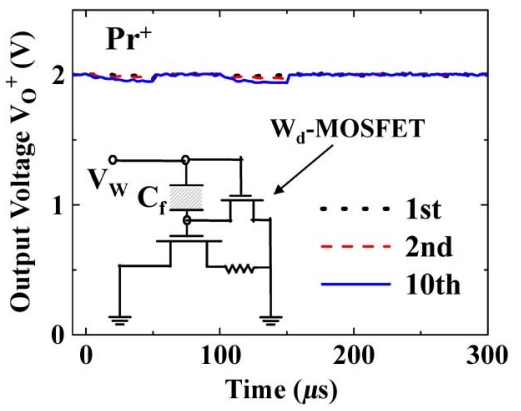

(a)

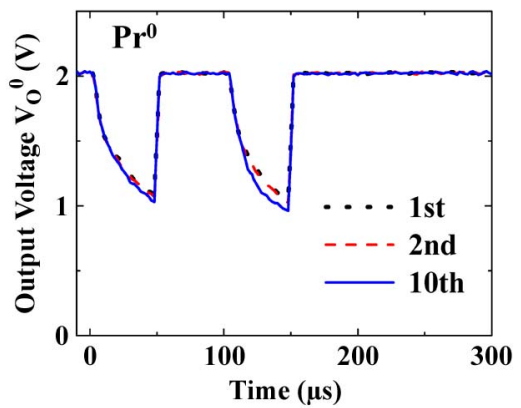

(b)

Fig. 6. Nondestructive readout characteristics for (a) the $\operatorname{Pr}^{+}$state and (b) the $\operatorname{Pr}^{0}$ state, using the writing pulse magnitudes of $V_{W}^{+}=4.0 \mathrm{~V}$ and $V_{W}^{-}=-2.3 \mathrm{~V}$, and the reading pulse magnitudes of $V_{R}^{+}=3.5 \mathrm{~V}$ and $V_{R}^{-}=-2.0 \mathrm{~V}$. The numbers of readout times are the first, second, and tenth, where the retention or interval time is 1 min after each readout. The $\mathrm{W}_{d}$-MOSFET was used only for writing $\mathrm{Pr}^{+}$memory state, which is not needed in integrated circuits.

without using $\mathrm{W}_{d}$-MOSFET. After experimentally confirming the nondestructive readout of the memory states written by the new writing operation, we investigated the write-disturbance characteristics.

\section{RESULTS AND Discussion}

Fig. 6(a) and (b) shows the nondestructive readout characteristics for the $\operatorname{Pr}^{+}$and $\operatorname{Pr}^{0}$ memory states, respectively. The memory state after each readout from the first to the tenth time was retained for 1 min under the condition that the IE was electrically floated and that the top electrode and the source and drain of the R-MOSFET were grounded. For every reading operation, the double periodic reading voltages were used. We can see that the characteristics for the $\operatorname{Pr}^{0}$ state, as well as those for the $\mathrm{Pr}^{+}$state, show almost the same as any readout time. Then, the output difference $\Delta V_{O}$ constantly remains approximately $1 \mathrm{~V}$ through all of the readout times. However, $V_{O}$ for the $\mathrm{Pr}^{+}$ and $\operatorname{Pr}^{0}$ states are decreased slightly with the readout times, and the $V_{O}$ for the second reading pulses are always lower than the first ones for any readout time. We can speculate possible causes for this phenomenon as follows: The negative pulse magnitude $V_{R}^{-}$of the reading voltage was adjusted so that the memory state could return to the initial memory state from the readout state by $V_{R}^{+}$. Because this adjustment was performed by only one readout time with one periodic reading voltage, the domain or polarization state of the $C_{f}$ might not become steady state, compared with readout memory states subjected to more readout operations. Therefore, some amount of negative domains or upward domains might be increased a little from the initial memory state by repeating the readout operation. Because we obtained nondestructive readout endurance over $10^{8}$ cycles as previously reported [16], it can be considered that the increment in volume of upward domain per one readout time is very small and is rapidly decreased with the number of readout times. Therefore, we can read out nondestructively the IF-FET memory written by the new writing operation. It is also noted that the response of $V_{O}$ is slow to the $V_{R}^{+}$pulse particularly in the $\operatorname{Pr}^{0}$ state. This is not due to the circuit time constant (i.e., $\left(C_{f} / / C_{i}\right) \cdot R$ ) because the time constant is approximately $0.2 \mu \mathrm{s}$ and much smaller than the pulsewidth of $50 \mu \mathrm{s}$. The main reason may be the requirement of some time to switch polarization domains, whose dynamics are governed by nucleation and growth of the ferroelectric domain [17]-[20]. It has been reported that the volume fraction $\Delta P(t) / \Delta P(\infty)=$ $p(t)$ of the switched domain, which is dependent of switching time $t$, can be described by $p(t)=1-\exp \left[\left(-t / t_{0}\right)^{n}\right] . \Delta P(t)$ is a switched polarization at $t$, and $\Delta P(\infty)$, which strongly depends on an electric field $E_{f}$ in $C_{f}$, is a saturation of switched polarization at infinite time. $t_{0}$ and $n$ are called the characteristics switching time and dimensionality, respectively, of the growing domains. It is well known that $t_{0}$ depends on $E_{f}$, crystal defect, grain size, electrode material, and so on. For example, Tagantsev et al. reported that $p(t)$ was approximately $80 \%$ at $t=1 \mathrm{~ms}$ for a 135 -nm-thick (111)-textured PZT film with an $\mathrm{IrO}_{x}$ top electrode and $E_{f}=100 \mathrm{kV} / \mathrm{cm}$ [17]. In our case, $E_{f}$ was approximately $100 \mathrm{kV} / \mathrm{cm}$, and the switching time was approximately $50 \mu \mathrm{s}$. Because $V_{I}$ is proportional to $\Delta P(t)$, it varies with $t$ corresponding to $p(t)$. Furthermore, by the transconductance $g_{m}$ of the R-MOSFET and $R$, an infinitesimal intermediate voltage or gate voltage $\delta V_{I}$ is amplified inversely to an infinitesimal output voltage $\delta V_{O}=$ $-\delta V_{I} \cdot g_{m} \cdot R$. Therefore, it was observed that the response of $V_{O}^{0}$ for $\operatorname{Pr}^{0}$ was fairly slow as shown in Fig. 6(b), compared with the $V_{R}^{+}$square pulse. If $E_{f}$ is increased by increasing $V_{R}^{+}$, we can obtain much faster response because it has been reported that $t_{0}$ follows an experimental low of $t_{0} \infty \exp \left(\alpha / E_{f}\right)$ [19], [21], where $\alpha$ is called as the activation field. Increasing $V_{R}^{+}$, however, probably leads to higher power consumption. Because ferroelectric films have a high potential of domain switching time of a few nanoseconds [22]-[24], an operation time of IF-FET can be reduced to nanosecond order by improving the material properties of the ferroelectric film and electrode and the process techniques.

Fig. 7(a) shows the write-disturbance characteristics of $\operatorname{Pr}^{0}$ memory state due to the previous writing operation. In this case, $V_{W}$ for $\operatorname{Pr}^{+}$is $4.0 \mathrm{~V}, V_{W}^{+}=4.0 \mathrm{~V}, V_{W}^{-}=-2.3 \mathrm{~V}, V_{R}^{+}=3.5 \mathrm{~V}$, and $V_{R}^{-}=-2.0 \mathrm{~V}$. From this figure, it can be seen that the output voltage $V_{O}^{0}$ for the $\operatorname{Pr}^{0}$ write disturbance remains almost constant during the measurement, which means that there is no write disturbance, as expected. However, the $V_{O}^{0}$ for the $\mathrm{Pr}^{+}$writing disturbance quickly increases with the number of writing times. $V_{O}^{0}$ of the initial memory state before the $\mathrm{Pr}^{+}$ write disturbance was approximately $0.9 \mathrm{~V}$, which was almost equal to $V_{O}^{0}$ of the $\operatorname{Pr}^{0}$ writing disturbance. This means that the memory state is destroyed and that severe write disturbance 


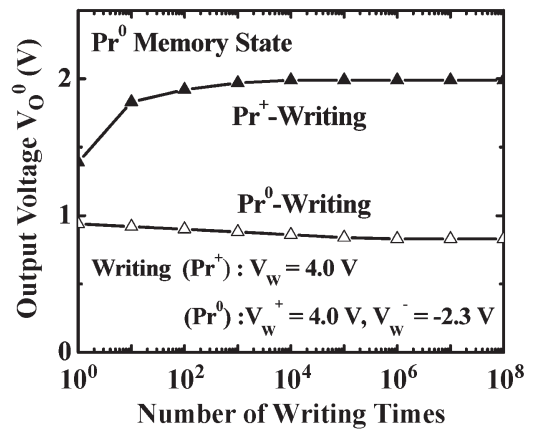

(a)

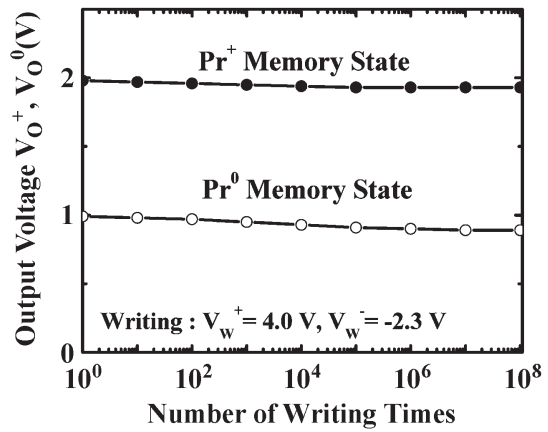

(b)

Fig. 7. (a) Write-disturbance characteristics of $\operatorname{Pr}^{0}$ memory state due to the previous writing operation, in which the writing voltages for $\operatorname{Pr}^{+}$and $\operatorname{Pr}^{0}$ states are different from each other. The $\mathrm{Pr}^{+}$write disturbance is severe, although the $\mathrm{Pr}^{0}$ write disturbance is almost free. (b) Write-disturbance characteristics of $\mathrm{Pr}^{+}$ and $\operatorname{Pr}^{0}$ memory states due to the writing operation proposed in this paper, in which the writing voltages for both states are the same.

occurs. This is because the positive writing voltages $V_{W}$ were applied continuously to the stored $\operatorname{Pr}^{0}$ memory state, which was changed toward a positive polarization from nearly neutral. For the $\mathrm{Pr}^{+}$memory state, write disturbance due to both the $\mathrm{Pr}^{+}$ and $\operatorname{Pr}^{0}$ writing operations was not observed.

Fig. 7(b) shows that there were no write disturbances due to the new writing operation for both memory states. Strictly speaking, the $V_{O}$ for both memory states are slightly decreased with the number of writing times. The same behavior is observed on $V_{O}^{0}$ due to the $\operatorname{Pr}^{0}$ writing disturbance shown in Fig. 7(a). This is because the polarizations of both the memory states may gradually change from the initial states according to the number of writing times in the final steady state, as shown in Fig. 6. The variations are so small to be negligible for the memory operation, and there is room to improve them further by adjusting the ferroelectric property of $C_{f}$ and the writing and reading pulse magnitudes [16]. Thus, from the results shown in Fig. 7(b), it can be said that the new writing operation is effective for write disturb free in IF-FET memory.

Finally, we discuss the integration issue of IF-FET. As previously mentioned, one ferroelectric capacitor (1C) of IF-FET can be formed directly on the gate oxide layer of R-MOSFET, the area of $1 \mathrm{C}$ being the same as that of the R-MOSFET gate. Under this condition of area, it is examined whether an operation voltage of IF-FET is reliable or not for commercialization. We estimate threshold reading voltages $V_{\mathrm{TR}}^{+}$for $\mathrm{Pr}^{+}$state and $V_{\mathrm{TR}}^{-}$for $\operatorname{Pr}^{0}$ state, at which $V_{I}$ is equal to $V_{\mathrm{th}}$ of R-MOSFET. They are given by the following simple formulas [14]:

$$
V_{\mathrm{TR}}^{+}=\left[\left(\frac{C_{i}}{C_{\mathrm{fl}}}\right)+1\right] V_{\mathrm{th}} \quad V_{\mathrm{TR}}^{-}=\left[\left(\frac{C_{i}}{C_{\mathrm{fh}}}\right)+1\right] V_{\mathrm{th}}
$$

The assumptions for the estimation are as follows: The gate structure is metal/100-nm-thick ferroelectric/metal/3-nm-thick $\mathrm{SiO}_{2}$ on $\mathrm{Si}$ in IF-FET, and $V_{\mathrm{th}}$ is $1.0 \mathrm{~V}$. The relative dielectric constants of $C_{i}, C_{\mathrm{fl}}$, and $C_{\mathrm{fh}}$ are 3.9, 200, and 400, respectively, according to the result shown in Fig. 5(b). Under this condition, we obtain $V_{\mathrm{TR}}^{+}=1.65 \mathrm{~V}$ and $V_{\mathrm{TR}}^{-}=1.33 \mathrm{~V}$. Because the difference between them is approximately $0.3 \mathrm{~V}$, and the operation voltage can be less than $1.5 \mathrm{~V}$, we can realize an IF-FET with the gate-stacked structure of metal/ferroelectric/metal/ $/ \mathrm{SiO}_{2}$. If a connection terminal of the IE is extended not from the edge of the source or drain but from the side in the channel width direction, self-align process may be available at the expense of a small additional area. Thus, the actual area of IF-FET cell in integrated configuration is occupied roughly by $2 \mathrm{~T}$. We can reduce the occupied area if the $2 \mathrm{~T}$ share the source or drain region in common. In static RAMs, thin-film transistors (TFTs) of polycrystalline $\mathrm{Si}$ are stacked over MOSFETs fabricated on a Si substrate to reduce the area per one memory cell [25]-[27]. If TFTs for W-MOSFETs are stacked over R-MOSFETs in integrated IF-FET, the packing density can be much increased. The drawback of IF-FET integration is technical complexity, such as fabrication of stacking TFTs over R-MOSFETs and the IEs between $C_{f}$ and $C_{i}$, which induces high cast. This problem may be eased somewhat if the stacked layers (e.g., TFT) are fabricated at temperature lower than $400{ }^{\circ} \mathrm{C}$. Low-temperature fabrication processes can reduce serious degradation of material properties (e.g., ferroelectric properties of $C_{f}$ ) in the under layers.

\section{CONClusion}

We have proposed a new operation for disturb-free writing in IF-FET memories. When $\mathrm{Pr}^{+}$and $\mathrm{Pr}^{0}$ states are written into memory cells, wordlines are applied by the same writing voltages consisting of a positive pulse magnitude $V_{W}^{+}$followed by a negative $V_{W}^{-}$, whereas the bitlines are applied by voltages that differ from each other. For the $\mathrm{Pr}^{+}$state, the bitline voltage falls down from high to low before the writing pulse magnitude changes from $V_{W}^{+}$to $V_{W}^{-}$. On the other hand, for the $\operatorname{Pr}^{0}$ state, the bitline voltage decreases when the whole writing voltage is finished. Because the waveform of the new writing voltage is similar to that of nondestructive reading voltage, IF-FET memory can be free from write disturbance. In fact, using the discrete circuit, we verified the usefulness of the new writing operation, showing disturb-free writing for any memory state for at least $10^{8}$ writing times. Furthermore, we discussed the possibility of the high integration of IF-FET and estimated the reading voltage that would be sufficient for the commercialization of IF-FET using a PZT film. To further improve write disturbance and nondestructive readout characteristics, better optimization on memory structure, ferroelectric properties, writing and reading pulse magnitudes, and bias timing of bitline, among others, would be required. 


\section{REFERENCES}

[1] J. F. Scott and C. A. Paz de Arauzo, "Ferroelectric memories," Science, vol. 246, no. 4936, pp. 1400-1405, Dec. 1989.

[2] H. Ishiwara, "Current status and prospects of FET-type ferroelectric memories," J. Semicond. Technol. Sci., vol. 1, no. 1, pp. 1-14, Mar. 2001.

[3] "International Technology Road Map for Semiconductors (ITRS)," pp. 8-10, 2005. [Online]. Available: http://www.itrs.net/Links/2005ITRS/ ERD2005.pdf

[4] S.-M. Yoon and H. Ishiwara, "Memory operation of 1T2C-type ferroelectric memory cell with excellent data retention characteristics," IEEE Trans. Electron Devices, vol. 48, no. 9, pp. 2002-2008, Sep. 2001.

[5] S. B. Xiong and S. Sakai, "Memory properties of $\mathrm{SrBi}_{2} \mathrm{Ta}_{2} \mathrm{O}_{9}$ thin film prepared on $\mathrm{SiO}_{2} / \mathrm{Si}$ substrates," Appl. Phys. Lett., vol. 75, no. 11, pp. 1613-1615, Sep. 1999.

[6] K.-H. Kim, J.-P. Han, S.-W. Jung, and T. P. Ma, "Ferroelectric DRAM (FEDRAM) FET with metal/ $/ \mathrm{SrBi}_{2} \mathrm{Ta}_{2} \mathrm{O}_{9} / \mathrm{SiN} / \mathrm{Si}$ gate structure," IEEE Electron Device Lett., vol. 23, no. 2, pp. 82-84, Feb. 2002.

[7] H. Saiki and E. Tokumitsu, "Ferroelectric split-gate-field-effecttransistors for nonvolatile memory cell array," IEICE Trans. Electron., vol. 87, no. 10, pp. 1700-1705, Oct. 2004.

[8] T. P. Ma and J.-P. Han, "Why is nonvolatile ferroelectric memory fieldeffect transistor still elusive?" IEEE Electron Device Lett., vol. 23, no. 7, pp. 386-388, Jul. 2002.

[9] C. T. Black, C. Farrell, and T. J. Licata, "Suppression of ferroelectric polarization by adjustable depolarization field," Appl. Phys. Lett., vol. 71, no. 14, pp. 2041-2043, Oct. 1997.

[10] B. Park, S. Shouriki, E. Tokumitsu, and H. Ishiwara, "Fabrication of $\mathrm{PbZr}_{x} \mathrm{Ti}_{1-x} \mathrm{O}_{3}$ films on $\mathrm{Si}$ structures using $\mathrm{Y}_{2} \mathrm{O}_{3}$ buffer layers," Jpn. J. Appl. Phys., vol. 37, no. 9B, pp. 5145-5149, Sep. 1998.

[11] T. Hirai, Y. Fujisaki, K. Nagashima, H. Koike, and Y. Tarui, "Preparation of $\mathrm{SrBi}_{2} \mathrm{Ta}_{2} \mathrm{O}_{9}$ film at low temperatures and fabrication of a metal/ferroelectric/insulator/semiconductor field effect transistor using $\mathrm{Al} / \mathrm{SrBi}_{2} \mathrm{Ta}_{2} \mathrm{O}_{9} / \mathrm{CeO}_{2} / \mathrm{Si}$ (100) structures," Jpn. J. Appl. Phys., vol. 36, no. 9B, pp. 5908-5911, Sep. 1997.

[12] S. Horita and T. D. Khoa, "Gate voltage reduction of a ferroelectric gate field effect transistor memory with an intermediate electrode on datareading," Jpn. J. Appl. Phys., vol. 42, no. 4A, pp. L365-L368, Apr. 2003.

[13] T. D. Khoa and S. Horita, "Retention and read endurance characteristics of a ferroelectric gate field-effect transistor memory with an intermediate electrode," Jpn. J. Appl. Phys., vol. 43, no. 4B, pp. 2220-2225, Apr. 2004.

[14] T. D. Khoa and S. Horita, "Analysis on operation of a F-FET memory with an intermediate electrode," IEEE Trans. Electron Devices, vol. 51, no. 5, pp. 820-823, May 2004

[15] B. N. Q. Trinh and S. Horita, "Operation of ferroelectric gate fieldeffect transistor memory with intermediate electrode using polycrystalline capacitor and metal-oxide-semiconductor field-effect transistor," Jpn. J. Appl. Phys., vol. 45, no. 9B, pp. 7341-7344, Sep. 2006.

[16] S. Horita and B. N. Q. Trinh, "Nondestructive readout of ferroelectric-gate field-effect transistor memory with an intermediate electrode by using an improved operation method," IEEE Trans. Electron Devices, vol. 55, no. 11, pp. 3200-3207, Nov. 2008.

[17] A. K. Tagantsev, I. Stolichnov, N. Setter, J. S. Cross, and M. Tsukada, "Non-Kolmogorov-Avrami switching kinetics in ferroelectric thin films," Phys. Rev. B, Condens. Matter, vol. 66, no. 21, pp. 214 109-1-214 109-6, Dec. 2002.

[18] I. Stolichnov, A. Tagantsev, N. Setter, J. S. Cross, and M. Tsukada, "Crossover between nucleation-controlled kinetics and domain wall motion kinetics of polarization reversal in ferroelectric films," Appl. Phys. Lett., vol. 83, no. 16, pp. 3362-3364, Oct. 2003.

[19] Y. W. So, D. J. Kim, T. W. Noh, J.-G. Yoon, and T. K. Song, "Polarization switching kinetics of epitaxial $\mathrm{Pb}\left(\mathrm{Zr}_{0.4} \mathrm{Ti}_{0.6}\right) \mathrm{O}_{3}$ thin films," Appl. Phys. Lett., vol. 86, no. 9, pp. 092 905-1-092905-3, Feb. 2005.

[20] A. Gruverman, B. J. Rodriguez, C. Dehoff, J. D. Waldrep, A. I. Kingon, R. J. Nemanich, and J. S. Cross, "Direct studies of domain switching dynamics in thin film ferroelectric capacitors," Appl. Phys. Lett., vol. 87, no. 8, pp. 082 902-1-082 902-3, Aug. 2005.
[21] W. J. Merz, "Domain formation and domain wall motions in ferroelectric $\mathrm{BaTiO}_{3}$ single crystals," Phys. Rev., vol. 95, no. 3, pp. 690-698, Aug. 1954.

[22] P. K. Larsen, G. L. M. Kampschöer, M. J. E. Ulenaers, G. A. C. M. Spierings, and R. Cuppens, "Nanosecond switching of thin ferroelectric films," Appl. Phys. Lett., vol. 59, no. 5, pp. 611-613, Jul. 1991.

[23] J. F. Scott, Ferroelectric Memories: Advanced Microelectronics. Berlin, Germany: Springer-Verlag, 2000

[24] A. Seike, K. Amanuma, S. Kobayashi, T. Tatsumi, H. Koike, and H. Hada, "Polarization reversal kinetics of a lead zirconate titanate thinfilm capacitor for nonvolatile memory," J. Appl. Phys., vol. 88, no. 6, pp. 3445-3447, Sep. 2000.

[25] S. Hayakawa, M. Kakumu, H. Takeuchi, K. Sato, T. Ohtani, T. Yoshida, T. Nakayama, S. Morita, M. Kinugawa, K. Maeguchi, K. Ochii, J. Matsunaga, A. Aono, K. Noguchi, and T. Asami, "A $1 \mu$ A retention 4Mb SRAM with a thin-film-transistor load cell," in Proc. ISSCC Tech. Dig., Feb. 1990, pp. 128-129.

[26] S. Ikeda, S. Hashiba, I. Kuramoto, H. Katoh, S. Ariga, T. Yamanaka, T. Hashimoto, N. Hashimoto, and S. Meguro, "A polysilicon transistor technology for large capacity SRAMs," in IEDM Tech. Dig., Dec. 1990, pp. 469-472.

[27] S. Ikeda, Y. Yoshida, S. Kamohara, K. Imato, K. Ishibashi, and K. Takahashi, "Threshold voltage-related soft error degradation in a TFT SRAM cell," IEEE Trans. Electron Devices, vol. 50, no. 2, pp. 391-396, Feb. 2003.

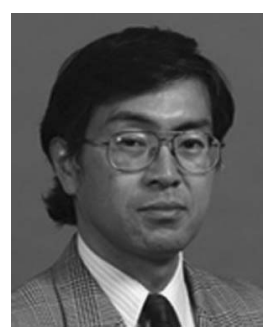

Susumu Horita was born in Toyama, Japan, in 1959. $\mathrm{He}$ received the B.S. degree in electrical engineering from Kanazawa University, Kanazawa, Japan, in 1982 and the M.S. and Ph.D. degrees in applied electronics from Tokyo Institute of Technology, Tokyo, Japan, in 1984 and 1987, respectively.

In 1987, he joined as a Research Associate with the Department of Electrical and Computer Engineering, Kanazawa University, where he become a Lecturer in 1988 and an Associate Professor in 1992. Since 1995, he has been an Associate Professor with the Graduate School of Materials Science, Japan Advanced Institute of Science and Technology, Nomi, Japan. His research interests are in the areas of ferroelectric memory, thin film transistors, and film formation process of electron device.

Dr. Horita is a member of the Materials Research Society, the Japan Society of Applied Physics, the Surface Science Society of Japan, and the Institute of Electronics Information and Communication Engineers.

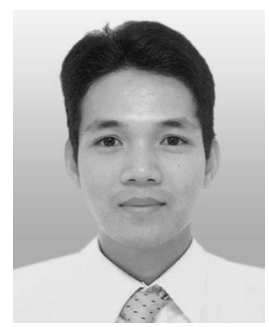

Bui Nguyen Quoc Trinh was born in Bacgiang City, Vietnam, in 1980. He received the B.S. and M.S. degrees in solid state physics from the Faculty of Physics, Hanoi University of Science, Vietnam National University, Hanoi, Vietnam, in 1998 and in 2004, respectively, and the Ph.D. degree from the Graduate School of Materials Science, Japan Advanced Institute of Science and Technology (JAIST), Nomi, Japan, in 2007.

He is currently with Prof. Shimoda as a Research Fellow in the Graduate School of Materials Science, JAIST. His research interests include characterizations of ferroelectric thin films, ferroelectric devices, and process technologies for Si-related devices.

Dr. Trinh is a member of the Japan Society of Applied Physics. 